\title{
Variabilité génétique de soja pour la résistance à la sclérotiniose (Sclerotinia sclerotiorum Lib de Bary). Liaisons statistiques avec certains caractères de la culture
}

\author{
J Gondran, P Leclercq \\ avec la collaboration de MF Pissard, A Faye \\ INRA, station d'amélioration des plantes fourragères, 86600 Lusignan, France
}

(Reçu le 18 juin 1991; accepté le 16 novembre 1992)

\begin{abstract}
Résumé - Pour de nombreux chercheurs, le classement des cultivars de soja pour la résistance à la sclérotiniose ne donne pas des résultats constants. Pour étudier ce problème, nous avons entrepris une expérimentation qui a duré 4 ans. Nous avons notamment mis en évidence que certains cultivars de soja, comme Labrador et Kingsoy, sont constamment et significativement moins attaqués que d'autres, tels que Dom et Messidor. Grâce à une technique d'infection par des sclérotes initiés, nous avons trouvé, sur les 31 cultivars étudiés, une corrélation significative entre la sensibilité à la sclérotiniose et la hauteur de la plante $(r=+0,54)$. Sur 17 cultivars les plus précoces parmi ces 31 , il existe une liaison du même ordre avec la date de maturité des gousses $(r=+0,57)$. Par contre, nous n'avons trouvé aucune corrélation significative avec d'autres caractères de la culture (date de fermeture du couvert végétal, date de floraison, nombre d'apothécies par $\mathrm{m}^{2}$, etc). Ces résultats sont discutés afin d'avoir une meilleure approche de la résistance du soja à la sclérotiniose.
\end{abstract}

Sclerotinia sclerotiorum / soja / résistance aux maladies / corrélation

Summary - Genetic variability of soybean for resistance to Sclerotinia sclerotiorum (Lib) de Bary. Statistical relationship with some traits of the crop. The ranking of the cultivars of soybean for stem rot resistance in many cases does not provide consistent results. To study this problem, we undertook a 4-yr experimental study. It was found that some cultivars such as Labrador and Kingsoy were less susceptible than others, eg Dom and Messidor. Using a new infection method of the soil via sclerotia, a significant correlation was found in 31 cultivars between the percentage of diseased plants and height at maturity $(r=+0.54)$. A significant correlation was also found with the susceptibility to stem rot and the date of pod maturity for the 17 earlier maturing cultivars $(r=+0.57)$. There was no significant correlation with the date of full canopy, the date of 50\% flowering, or the number of apothecia per $\mathrm{m}^{2}$. These results are discussed and a viable approach regarding resistance of soybean to S sclerotiorum is proposed.

\section{Sclerotinia sclerotiorum / soybean / disease resistance / correlation}

\footnotetext{
* Correspondance et tirés à part
} 


\section{INTRODUCTION}

La sclérotiniose est la plus grave maladie du soja dans les régions du monde correspondant aux limites septentrionales de cette culture : Amérique du Nord, Chine du Nord et Europe (Grau et Radke, 1984; Chun et al, 1987; Boland et Hall, 1988; Li Hanching et Fu Chunyan, 1981; Ganya, 1981).

La récolte en graine peut être réduite dans des proportions supérieures à $40 \%$ (Grau et Radke, 1984; Chun et al, 1987; Gondran et Leclerc, 1991).

Dans nos climats, les symptômes de flétrissements causés par $S$ sclerotiorum peuvent apparaître dans les champs de soja 15 j après la fermeture du couvert végétal (interligne cessant de recevoir au sol la lumière directe du soleil) (figs 1 et 2). Sur les tiges atteintes, on peut remarquer des sclérotes, d'abord blancs puis noirs (fig 5). Ces organes permettent la conservation dans le sol du champignon vivant durant 4 à 7 ans (Purdy, 1979). Ils se forment le plus souvent à l'intérieur de la cavité médullaire des tiges de soja (Gondran et Leclercq, 1990). L'année qui suit la récolte, ces sclérotes différencient à la surface du sol des apothécies (figs 3 et 4) émétrices d'ascospores, qui sont responsables des infections. S sclerotiorum infecte plus de 380 espèces de plantes, dont de mauvaises herbes et d'importantes cultures : tournesol, colza, pois, tabac, lupins, etc (Purdy, 1979). L'extention depuis 15 ans des cultures sensibles de tournesol, pois et colza, rend donc ce champignon phytopathogène encore plus redoutable. En effet, la multiplication et l'accumulation des sclérotes dans le sol augmentent chaque année. On peut estimer que dans 20 ans, plus du quart des terres labourées françaises sera infesté par des sclérotes de $S$ sclerotiorum. Soulignons que

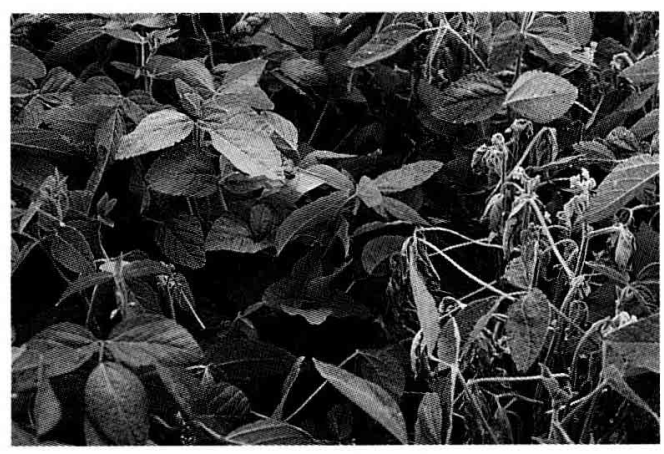

Fig 1. Flétrissement causé par $S$ sclerotiorum dans une parcelle de soja.

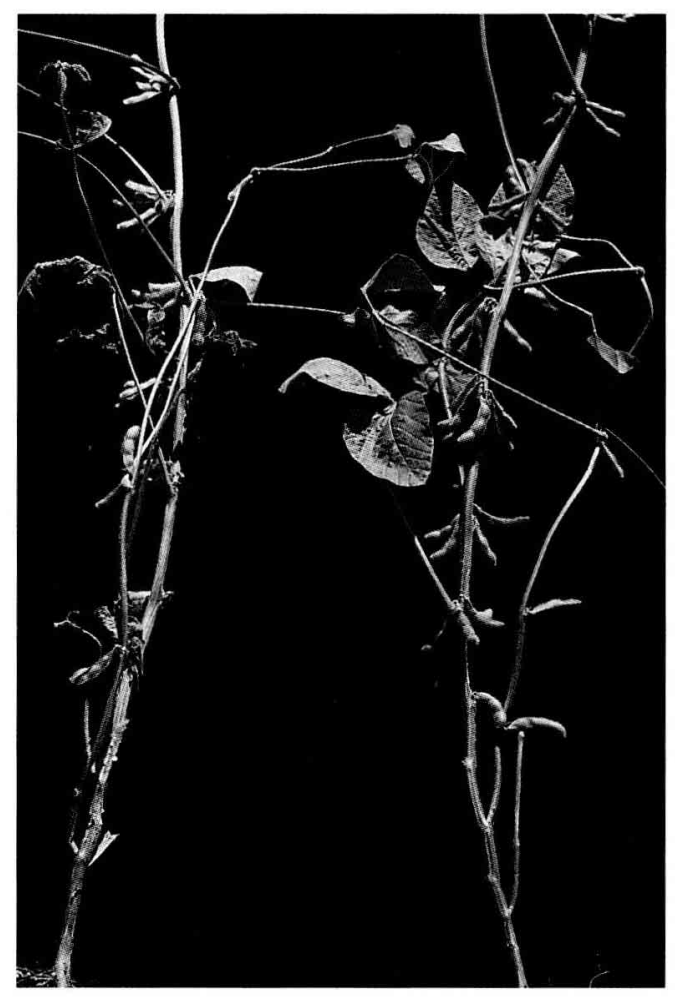

Fig 2. À gauche, une plante flétrie à la suite d'une attaque de $S$ sclerotiorum. À droite, une plante saine au même stade de développement.

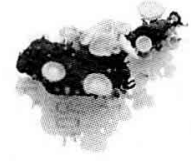

Fig 3. Apothécies se formant sur un sclérote.

cette extention probable n'est valable que pour l'Europe.

La lutte chimique est quelquefois recommandée (Penaud, 1991). Mais nous pensons qu'elle est trop aléatoire. Par ailleurs, elle nous paraît actuellement trop onéreuse pour l'agriculteur, du fait de la nouvelle politique agricole commune, qui diminue beaucoup le revenu brut des cultures. Enfin, la formation et l'accumulation des résidus rend l'utilisation de la lutte chimique de plus en plus difficile.

Les études réalisées depuis 10 ans par les chercheurs américains pour trouver une variabili- 


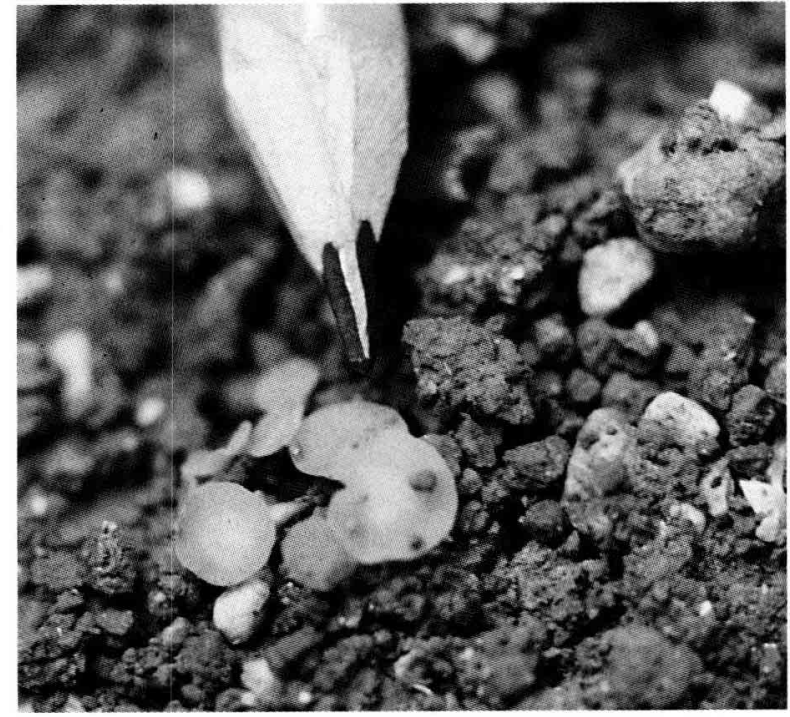

Fig 4. Apothécie se formant à la surface d'un sol infesté.

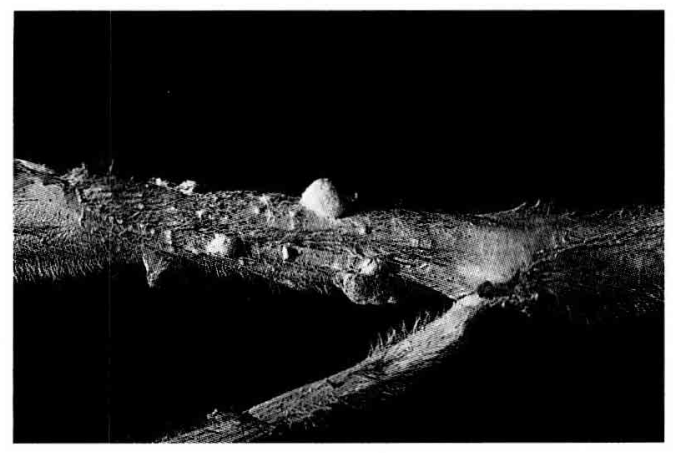

Fig 5. Sclérotes sur une tige de soja attaquée par la sclérotinose.

té génétique pour la résistance, ont abouti à mettre en évidence certains cultivars plus résistants que d'autres. Mais cette variabilité nous paraît faible (Nelson et al, 1991a) et donne rarement des résultats constants (Nelson et al, 1991b). Nous avons donc entrepris une étude pour rechercher une plus large variabilité entre les cultivars ou obtentions de soja, pour essayer d'obtenir des classements constants et pour tenter d'expliquer cette résistance par des liaisons avec d'autres caractères de la culture de soja.

\section{MATÉRIEL ET MÉTHODES}

Des cultivars de soja appartenant aux groupes de précocité ooo à I (tableau I) et d'autres obtentions et va- riétés appartenant aux groupes plus tardifs (tableau II) sont semés dans les conditions naturelles à la densité de 500000 graines/ha.

Le sol est naturellement ou artificiellement infecté par des sclérotes de $S$ sclerotiorum.

\section{Sols naturellement infestés}

\section{Essais réalisés à Savarit en 1988-1990}

Dans la parcelle d'un agriculteur des CharentesMaritimes, mis à notre disposition par le CETIOM, le soja a été précédé par une culture de tournesol très atteinte par $S$ sclerotiorum. De cette façon, de nombreux sclérotes sont présents dans le sol, et nous pouvions espérer une infection homogène du soja dans les conditions naturelles. En 1988, la surface de chaque parcelle était de $14,4 \mathrm{~m}^{2}$. II y avait 7 variétés réparties en 4 blocs. En 1989 et en 1990, 12 cultivars étaient semés dans des parcelles élémentaires de $22 \mathrm{~m}^{2}$ disposées en 3 blocs. Afin de favoriser la maladie, des arrosages par aspersion sont réalisés sitôt l'apparition des premières apothécies. Les notations de maladie ont lieu les 31 août 1988, 30 août 1989 et 25 août 1990. Les plantes malades situées sur la ligne médiane de la parcelle élémentaire, soit sur une longueur de $8 \mathrm{~m}$ pour l'essai de 1988 , et une longueur de $12 \mathrm{~m}$ pour les essais de 1989 et 1990, sont dénombrées. Les plantes atteintes de sclérotiniose sont aisément distinguées des plantes saines par la couleur plus claire de l'épiderme et par la présence de sclérotes à l'intérieur de la cavité médullaire des tiges (Gondran et Leclercq, 1990).

Les résultats sont exprimés en pourcentage de plantes malades par rapport aux plantes dénombrées sur la ligne médiane de la parcelle.

\section{Essais réalisés à Villemur en 1989 , à Montauban en 1990 et à Agen en 1991}

Les sclérotes infestant le sol proviennent d'un précédent de culture de soja malade. Les arrosages, toujours par aspersion, commencent début juin, sitôt l'apparition des premières apothécies, et sont poursuivis jusqu'à la fin août.

Dans le premier essai installé dans le domaine de Carles à Villemur (31), appartenant à la firme Protosem, les parcelles ont une surface de $120 \mathrm{~m}^{2}$. Les notations sont réalisées le 12 sept 1989 en dénombrant le pourcentage de plantes malades sur la ligne centrale de la parcelle qui a $60 \mathrm{~m}$ de long.

Le terrain de l'essai situé en 1990 à Montauban (82) avait été loué à un agriculteur par la firme Rustica-Semences. Chaque parcelle avait une surface de $13,5 \mathrm{~m}^{2}$. La notation a été réalisée le 12 sept 1990 en dénombrant des plantes malades sur la longueur de $7,5 \mathrm{~m}$ de la ligne centrale de chaque parcelle. 
Tableau I. Infection par la sclérotiniose de cultivars précoces de soja.

\begin{tabular}{|c|c|c|c|c|c|c|c|}
\hline \multirow[t]{2}{*}{ Cultivars } & \multirow{2}{*}{$\begin{array}{l}\text { Groupe de } \\
\text { précocité }\end{array}$} & \multicolumn{6}{|c|}{ Pourcentage de plantes malades } \\
\hline & & $\begin{array}{c}1988 \\
\text { Savarit (N) }\end{array}$ & $\begin{array}{c}1989 \\
\text { Savarit (N) }\end{array}$ & $\begin{array}{c}1990 \\
\text { Savarit (N) }\end{array}$ & $\begin{array}{c}1990 \\
\text { Lusignan (A) }\end{array}$ & $\begin{array}{c}1991 \\
\text { Agen (N) }\end{array}$ & $\begin{array}{c}1991 \\
\text { Lusignan }(A)\end{array}$ \\
\hline Chandor & 0 & $51^{b}$ & $3^{a}$ & $0^{a}$ & $4^{a}$ & $77^{a}$ & - \\
\hline Pelvoux & 00 & - & - & 0 a & $6 a$ & - & - \\
\hline Maple-Arrow & 00 & 28 a & - & $0^{a}$ & $6 a$ & - & - \\
\hline Maple-Glen & 00 & - & $2^{a}$ & - & $8^{a}$ & - & - \\
\hline Ultra & 000 & - & - & - & $10 a$ & - & - \\
\hline Labrador & 00 & 28 a & $0^{a}$ & $0^{a}$ & $10 \mathrm{a}$ & - & $13^{a}$ \\
\hline Effi & 00 & - & - & $0^{a}$ & $15 \mathrm{ab}$ & - & - \\
\hline Kalmit & 00 & - & $4^{a}$ & $0^{a}$ & $25 a b c$ & - & - \\
\hline Apache & $0-00$ & - & $2^{a}$ & $0^{a}$ & $40 \mathrm{bcd}$ & - & - \\
\hline Cervin & 00 & - & - & $0 \mathrm{a}$ & $40 \mathrm{bcd}$ & - & - \\
\hline Perla & $0-1$ & - & $6^{a}$ & - & 45 cde & - & - \\
\hline Dawson & 0 & $54 \mathrm{~b}$ & $1 \mathrm{a}$ & $3^{b}$ & $54 d c$ & - & - \\
\hline Sakura & 0 & - & $2^{a}$ & - & 63 de & - & - \\
\hline Crusader & 0 & - & $4^{a}$ & - & 65 de & - & - \\
\hline Swift & 0 & $55^{b}$ & $6^{a}$ & $1 \mathrm{a}$ & $71 \mathrm{e}$ & - & - \\
\hline Weber & I & $60^{b}$ & $22^{b}$ & $5^{c}$ & $72 \mathrm{e}$ & - & - \\
\hline Dom & 0 & $62^{b}$ & 5 a & $3^{b}$ & $74 \mathrm{e}$ & $75^{a}$ & $48^{b}$ \\
\hline \multirow[t]{2}{*}{ mm d'eau } & (irrigations) & $157^{\star}$ & $135^{\star}$ & $160^{*}$ & $795^{\star}$ & $145^{\star *}$ & $312^{*}$ \\
\hline & (pluies) & $120^{\star}$ & $73^{\star}$ & $81^{*}$ & $37^{*}$ & $161^{\star *}$ & $55^{\star}$ \\
\hline
\end{tabular}

* Durant les mois de juillet et août; ** durant les mois de juin, juillet et août; $N$ : infection naturelle dans les conditions naturelles; $A$ : infection artificielle par sclérotes dans des conditions naturelles. $a, b, c, d, e$ : différences significatives selon le test de Duncan $(P<$ $0,05)$.

À Agen (47) en 1991, le CETIOM avait réalisé un essai qui comprenait 7 variétés de soja réparties en 4 blocs. Les plantes malades et saines ont été comptées sur une longueur de $8 \mathrm{~m}$ le 2 sept 1991 sur la ligne centrale de chaque parcelle, dont la surface était de $36 \mathrm{~m}^{2}$.

\section{Sols artificiellement infestés}

Pour mieux surveiller le développement de la maladie et celui des plantes, nous avons installé à Lusignan à proximité de notre Laboratoire un essai en 1990 et un autre en 1991. Comme le sol était dépourvu de sclérotes de $S$ sclerotiorum, nous avons expérimenté le procédé d'infection suivant. Des sclérotes récoltés sur tournesol malade sont induits par un séjour préalable sous $5 \mathrm{~cm}$ de terre dans les conditions extérieures en période froide (de novembre à avril), selon la méthode de Lamarque (1976). Les sclérotes ainsi induits sont épandus 1 mois après la levée, sur une longueur de $2 \mathrm{~m}$ à $5 \mathrm{~cm}$ de la ligne centrale. Ils sont recouverts de terre. Nous avons utilisé $44 \mathrm{~g}$ de sclérotes secs par parcelle élémentaire en 1990 et $60 \mathrm{~g}$ en 1991. Ces parcelles sont constituées par 3 lignes de $4 \mathrm{~m}$ de long espacées de $40 \mathrm{~cm}$, soit une surface de $4,8 \mathrm{~m}^{2}$. Trois répétitions sont réalisées. Les obtentions et cultivars de soja comparés s'élevaient à 31 en 1990 et ont été réduits au nombre de 4 en 1991. En effet, nous avons choisi en 1991 ceux qui s'étaient révélés très différents des années précédentes pour leur résistance à la sclérotiniose.

Les notations sont faites le 17 sept 1990 pour le $1^{\text {er }}$ essai et le 24 sept 1991 pour le $2^{\mathrm{e}}$ en dénombrant le nombre de plantes atteintes de sclérotiniose sur la longueur de $2 \mathrm{~m}$ de la ligne centrale ayant reçu auparavant les sclérotes. Les résultats sont exprimés en pourcentage de plantes malades par rapport au nombre total de plantes comptées sur cette longueur de ligne.

En 1990, de nombreuses caractéristiques de la plante et de la culture sont notées (tableaux III, IV, V). Ce sont : la date de fermeture du couvert végétal, la hauteur de la plante à la floraison, la longueur de la $2^{\mathrm{e}}$ feuille trifoliée le 6 juin 1990, le nombre de ramifications à la floraison, la date de la 1re fleur, la date de floraison, le nombre d'apothécies par $\mathrm{m}^{2}$, la date de maturité des gousses, la hauteur de la plante à la maturité. L'étude de leur liaison statistique avec le pourcentage de plantes malades est réalisée en calculant les coefficients de corrélation. 
Tableau II. Infection par la sclérotiniose de cultivars tardifs de soja.

\begin{tabular}{|c|c|c|c|c|c|c|}
\hline \multirow[t]{2}{*}{ Cultivars } & \multirow{2}{*}{$\begin{array}{l}\text { Groupe de } \\
\text { précocité }\end{array}$} & \multicolumn{5}{|c|}{ Pourcentage de plantes malades } \\
\hline & & $\begin{array}{c}1989^{\star * *} \\
\text { Villemur }(N)\end{array}$ & $\begin{array}{c}1990 \\
\text { Montauban (N) }\end{array}$ & $\begin{array}{c}1990 \\
\text { Lusignan }(A)\end{array}$ & $\begin{array}{c}1991 \\
\text { Agen }(N)\end{array}$ & $\begin{array}{c}1991 \\
\text { Lusignan (A) }\end{array}$ \\
\hline Kingsoy & II & 0 & $18^{a b}$ & $24^{a}$ & $62^{a b}$ & $13^{a}$ \\
\hline Exp 2 & II & - & $12^{a}$ & $25^{a b}$ & - & - \\
\hline Frisco & II & - & $26 a b c$ & $31 a b$ & - & - \\
\hline Alaric & II & 5 & $30 \mathrm{abcd}$ & $33 a b$ & - & - \\
\hline Argenta & I & - & 26 abcd & $43 a b c$ & 91 bc & - \\
\hline Spot & 1 & - & $16 a b$ & $46 \mathrm{abc}$ & - & - \\
\hline Fuji & I & 6 & $20 \mathrm{ab}$ & $50 a b c$ & - & - \\
\hline Canton & 1 & 0 & $31 \mathrm{abcd}$ & $60 \mathrm{abcd}$ & $60^{a}$ & - \\
\hline $15-7$ & 1 & 4 & $20 \mathrm{ab}$ & 62 abcd & - & - \\
\hline Eszter & 1 & 3 & $32 \mathrm{abcd}$ & $65 \mathrm{abcd}$ & - & - \\
\hline Exp 1 & 1 & - & $47 \mathrm{~cd}$ & $65^{\text {abcd }}$ & - & - \\
\hline Kushiro & I & - & $50 \mathrm{~cd}$ & $66 \mathrm{bcd}$ & $92^{c}$ & - \\
\hline Messidor & 1 & 7 & $40 \mathrm{bcd}$ & $93^{d}$ & - & $25^{b}$ \\
\hline Kador & II & - & $31 \mathrm{abcd}$ & $76 \mathrm{~cd}$ & $76^{b c}$ & - \\
\hline Weber & 1 & 0 & $53^{d}$ & $72 \mathrm{~cd}$ & - & - \\
\hline \multicolumn{7}{|l|}{ mm d'eau } \\
\hline Irrigations & & $270^{\star \star}$ & $270^{\star \star}$ & $795^{*}$ & $145^{\star \star}$ & $312^{*}$ \\
\hline Pluies & & $91^{\star \star}$ & $122^{\star}$ & $37^{\star}$ & $161^{\star \star}$ & $55^{\star}$ \\
\hline
\end{tabular}

* Durant les mois de juillet et d'août; ** durant les mois de juin, juillet et août; ${ }^{* * *}$ résultats non significatifs par le test de $F(P<0,05)$; $\mathrm{N}$ : infection naturelle dans les conditions naturelles; $A$ : infection artificielle par scléortes dans les conditions naturelles; $a, b, c, d:$ différence significative $(P<0,05)$ par le test de Duncan.

\section{RÉSULTATS}

\section{Différences de sensibilité pour la résistance à la sclérotiniose des cultivars du soja}

Les pourcentages de plantes malades notées sur les cultivars précoces sont rassemblés dans le tableau I. Ceux concernant les obtentions plus tardives sont représentés dans le tableau II. Dans les 2 cas, nous constatons que certaines variétés sont très différentes pour leur résistance à la sclérotiniose. Ainsi, Labrador est toujours moins attaqué que Dom et Kingsoy est classé plus résistant que Messidor.

En revanche, le cultivar Chandor n'a pas un classement constant. II est sensible à Savarit en 1988 et à Agen en 1991. Il est résistant à Lusignan en 1990. Le caractère artificiel de l'infection pourrait être responsable de cette différence de classement.

Notons que pour certains essais, l'infection est très faible (Savarit, 1989 et 1990; Villemur, 1989).
Ces faibles attaques sont explicables par une humidité insuffisante pour le développement des apothécies et pour la contamination par les ascospores. En effet, à Villemur le sol était caillouteux et très filtrant. L'eau restait peu de temps à la surface du sol pour permettre le développement des apothécies qui étaient trop rares. L'infection a été fortement hétérogène et les différences variétales n'ont pu être décelées (test de $F$ non significatif). À Savarit en 1989 et 1990, par suite des pannes dans le système d'irrigation, les apports d'eau ont été insuffisants. Nous pouvons dire qu'un terrain peu filtrant et que l'apport en juin, juillet et août de $300 \mathrm{~mm}$ d'eau sont nécessaires pour assurer une infection permettant de classer les cultivars de soja pour leur résistance à la sclérotinose.

La méthode d'infection du sol par l'apport de sclérotes induits est efficace, puisque les infections sont en bonne correspondance avec les infections naturelles. Notamment, les coefficients de corrélation entre le classement des variétés lors de l'infection artificielle à Lusignan en 1990 et des infections artificielles dans différents lieux et années sont : 
Tableau III. Résistance du soja à la sclérotiniose pouvant être expliquée par plusieurs caractères (essai à Lusignan en 1990).

\begin{tabular}{cccccc}
\hline Cultivars & $\begin{array}{c}\text { Pourcentage de } \\
\text { plantes malades }\end{array}$ & $\begin{array}{c}\text { Hauteur en } \mathrm{cm} \\
\text { à la maturité } \\
\text { des gousses }\end{array}$ & $\begin{array}{c}\text { Date de } \\
\text { maturité } \\
\text { des gousses * }\end{array}$ & $\begin{array}{c}\text { Couleur } \\
\text { des } \\
\text { fleurs }\end{array}$ & $\begin{array}{c}\text { Résidus indépendants } \\
\text { de la hauteur } \\
\text { et de la date }\end{array}$ \\
de maturité de gousses
\end{tabular}

\begin{tabular}{|c|c|c|c|c|c|}
\hline Chandor & $4^{a}$ & 120 & 24 & $\mathrm{~B}$ & $-42 a$ \\
\hline Pelvoux & $6^{a}$ & 103 & 17 & V & $-19 a b c$ \\
\hline Maple-Arrow & $6^{a}$ & 120 & 17 & V & $-36 a b$ \\
\hline Maple-Glen & $7^{a b}$ & 107 & 17 & V & $-21 a b c$ \\
\hline Ultra & $10 a b c$ & 100 & 5 & V & $-4 a b c$ \\
\hline Labrador & $10 a b c$ & 108 & 18 & V & $-21 a b c$ \\
\hline Effi & $15^{\text {abcd }}$ & 110 & 12 & V & $-16 a b c$ \\
\hline Kingsoy & 24 abcde & 130 & 39 & V & $-42 \mathrm{a}$ \\
\hline Kalmit & 25 abcde & 112 & 11 & V & $-5 a b c$ \\
\hline Exp 2 & 25 abcde & 128 & 41 & $\mathrm{~B}$ & $-40 a$ \\
\hline Frisco & 31 abcde & 115 & 28 & V & $-13 a b c$ \\
\hline Alaric & 33 abcdef & 102 & 28 & V & -3 acc \\
\hline Apache & 39 abcdef & 115 & 11 & V & $+7 a b c$ \\
\hline Cervin & 40 abcdef & 108 & 12 & V & $+15^{a b c}$ \\
\hline Argenta & 43 abcdef & 128 & 32 & V & $-16 a b c$ \\
\hline Spot & 46 abcdef & 110 & 36 & V & $+2 a b c$ \\
\hline Perla & 48 abcdef & 130 & 20 & V & $-9 a b c$ \\
\hline Fuji & 50 abcdef & 122 & 31 & V & $-3 a b c$ \\
\hline Dawson & 54 abcdef & 120 & 15 & V & $+13 a b c$ \\
\hline Canton & 60 bcdef & 122 & 31 & V & $-4 a b c$ \\
\hline 15.7 & 62 bcdef & 133 & 25 & V & $+2 a b c$ \\
\hline Sakura & 63 cdef & 130 & 27 & $B$ & $+4 a b c$ \\
\hline Eszter & 65 cdef & 112 & 25 & V & $+26^{c}$ \\
\hline Crusader & $65^{\text {cdef }}$ & 120 & 22 & $\mathrm{~B}$ & $+20 b c$ \\
\hline Exp 1 & 65 cdef & 115 & 39 & B & $+15 a b c$ \\
\hline Kushiro & 66 def & 105 & 24 & $\mathrm{~V}$ & $+35 c$ \\
\hline Swift & 71 ef & 132 & 24 & $\mathrm{~B}$ & $+12 a b c$ \\
\hline Weber & 72 ef & 127 & 35 & $\mathrm{~B}$ & $+12 a b c$ \\
\hline Dom & 73 ef & 125 & 24 & $B$ & $+22^{c}$ \\
\hline Kador & 76 ef & 125 & 32 & V & $+20 b c$ \\
\hline Messidor & $93^{f}$ & 140 & 20 & V & $+29 c$ \\
\hline
\end{tabular}

* En jours à partir du $1^{\text {er }}$ septembre 1990; a, b, c, d, e, f : différences significatives par le test de Newman-Keuls $(P<0,05)$; B : fleurs blanches; $V$ : fleurs violettes.

- Savarit, $1988: r=+0,81(P<0,05)$;

- Savarit, $1989: r=+0,76(P<0,01)$;

- Montauban, $1990: r=+0,69(P<0,01)$.

\section{Caractères de la culture de soja pouvant expliquer la résistance à la sclérotiniose}

Dans l'essai réalisé à Lusignan en 1990, nous remarquons que la date de maturité des gousses et la hauteur de la plante à la maturité sont liées significativement à la sensibilité de la plante, sur- tout pour les cultivars plus précoces (tableaux III et $V$ ). Nous avons calculé l'équation de régression double avec les variables suivantes : $Y$ : pourcentage de plantes atteintes par la sclérotiniose; $X$ : hauteur des plantes à la maturité; $Z$ : date de maturité des gousses. Nous trouvons:

$$
Y=1,01 X+0,63 Z-90,18 .
$$

Nous pouvons donc calculer le résidu statistique $R$ :

$$
R=Y-1,01 X-0,63 Z+90,18
$$


Tableau IV. Données de la culture de soja n'expliquant pas la résistance à la sclérotiniose (Lusignan 90).

\begin{tabular}{|c|c|c|c|c|c|c|c|c|c|}
\hline \multirow[t]{2}{*}{ Cultivars } & \multirow{2}{*}{$\begin{array}{c}\text { Date de } \\
\text { de fermeture } \\
\text { couvert végétal }\end{array}$} & \multirow{2}{*}{$\begin{array}{c}\text { Hauteur } \\
\text { plante } \\
\text { à la floraison } \\
\text { (en } \mathrm{cm})\end{array}$} & \multirow{2}{*}{$\begin{array}{c}\text { Longueur } \\
2^{e} \text { feuille } \\
\text { trifoliée } \\
\text { (en cm) } \\
6 \text { juin } 1990\end{array}$} & \multirow{2}{*}{$\begin{array}{l}\text { Nombre } \\
\text { ramification } \\
\text { à la } \\
\text { floraison }\end{array}$} & \multirow{2}{*}{$\begin{array}{l}\text { Nombre } \\
\text { ramification } \\
\text { à maturité }\end{array}$} & \multirow{2}{*}{$\begin{array}{c}\text { Date } \\
\text { de la } \\
\text { yre fleur }\end{array}$} & \multirow{2}{*}{$\begin{array}{c}\text { Date } \\
\text { de } \\
\text { floraison }\end{array}$} & \multicolumn{2}{|c|}{$\begin{array}{c}\text { Nombre } \\
\text { d'apothécies }\end{array}$} \\
\hline & & & & & & & & $\begin{array}{r}1988 \\
\text { Savarit }\end{array}$ & $\begin{array}{c}1990 \\
\text { Lusignan }\end{array}$ \\
\hline Chandor & 31 & 47 & 13 & 1,9 & 1,2 & 22 & 28 & 4 & 43 \\
\hline Pelvoux & 39 & 50 & 13 & 2,1 & 1,5 & 22 & 29 & - & 143 \\
\hline Maple-Arow & 36 & 46 & 14 & 1,3 & 1,1 & 19 & 27 & 8 & 90 \\
\hline Maple-Glen & 43 & 37 & 13 & 1,2 & 1,3 & 19 & 25 & - & 224 \\
\hline Ultra & 34 & 47 & 13 & 0,8 & 0,4 & 20 & 27 & - & 43 \\
\hline Labrador & 44 & 44 & 11 & 2,1 & 1,4 & 18 & 25 & 4 & 53 \\
\hline Effi & 27 & 95 & 14 & 1,7 & 1,1 & 33 & 46 & - & 239 \\
\hline Kingsoy & 28 & 93 & 16 & 3,0 & 3,1 & 53 & 55 & - & 29 \\
\hline Kalmit & 36 & 66 & 13 & 2,3 & 1,1 & 23 & 32 & - & 300 \\
\hline Exp 2 & 38 & 75 & 16 & 3,0 & 2,4 & 55 & 55 & - & 43 \\
\hline Frisco & 29 & 67 & 14 & 2,0 & 1,7 & 28 & 39 & - & 153 \\
\hline Alaric & 27 & 77 & 18 & 2,5 & 2,7 & 47 & 55 & - & 43 \\
\hline Apache & 46 & 41 & 12 & 1,1 & 0,5 & 21 & 28 & - & 186 \\
\hline Cervin & 36 & 44 & 12 & 0,8 & 0,6 & 20 & 28 & - & 96 \\
\hline Argenta & 33 & 52 & 15 & 3,1 & 2,2 & 24 & 32 & - & 329 \\
\hline Spot & 39 & 77 & 17 & 2,2 & 2,4 & 51 & 53 & - & 61 \\
\hline Perla & 33 & 43 & 12 & 1,9 & 1,9 & 27 & 29 & - & 81 \\
\hline Fuji & 23 & 50 & 14 & 0,8 & 0,6 & 26 & 30 & - & 76 \\
\hline Dawson & 33 & 48 & 14 & 1,5 & 1,0 & 23 & 28 & 3 & 171 \\
\hline Canton & 32 & 80 & 13 & 0,9 & 1,1 & 29 & 46 & & 14 \\
\hline 15.7 & 33 & 68 & 15 & 3,7 & 3,3 & 47 & 55 & & 143 \\
\hline Sakura & 43 & 54 & 11 & 1,5 & 1,5 & 27 & 32 & - & 200 \\
\hline Eszter & 32 & 52 & 16 & 1,5 & 2,1 & 24 & 29 & - & 47 \\
\hline Crusader & 35 & 48 & 10 & 1,0 & 0,6 & 25 & 28 & - & 43 \\
\hline Exp 1 & 42 & 95 & 16 & 2,3 & 3,6 & 50 & 53 & - & 114 \\
\hline Kushiro & 23 & 58 & 15 & 1,9 & 1,7 & 29 & 34 & - & 147 \\
\hline Swift & 32 & 89 & 15 & 1,7 & 0,9 & 25 & 44 & 4 & 261 \\
\hline Weber & 43 & 89 & 10 & 2,1 & 1,6 & 29 & 50 & 5 & 0 \\
\hline Dom & 40 & 46 & 13 & 1,8 & 1,5 & 25 & 28 & 5 & 14 \\
\hline Kador & 43 & 89 & 14 & 2,7 & 3,8 & 36 & 55 & - & 90 \\
\hline Messidor & 43 & 10 & 15 & 2,3 & 1,7 & 28 & 47 & - & 61 \\
\hline
\end{tabular}

* Sur une portion de la surface de parcelles de $2,8 \mathrm{~m}^{2} ;{ }^{\star \star}$ sur une portion de la surface de la parcelle de $0,07 \mathrm{~m}^{2}$.

Cette valeur $R$ correspond à la sensibilité à la sclérotiniose après élimination des effets concernant à la fois la hauteur des plantes à maturité et aux dates de maturité des gousses. Les 31 variétés de soja sont donc classées suivant des grandeurs négatives, si les variétés sont plus résistantes que la droite de régression double l'indique, et positives si elles sont plus sensibles que cette droite l'exprime (tableau III).

Nous avons calculé le coefficient de correlation $r$, entre le pourcentage de plantes malades de sclérotiniose et le résidu statistique :

$$
r=+0,85(P<0,001) \text {. }
$$

La résistance à la sclérotiniose des 31 variétés est donc largement exprimée par une valeur qui ne dépend ni de la hauteur des plantes à maturité, ni de la date de maturité des gousses.

Nous nous sommes alors demandé si la couleur des fleurs pouvait expliquer la sensibilité des plantes à la sclérotiniose. En effet, dans le tableau III, nous pouvons remarquer que les variétés à fleurs blanches sont plus fréquemment sensibles que les variétés à fleurs violettes qui 
Tableau V. Coefficient de corrélation entre le pourcentage des plantes malades et divers autres caractères de la culture de soja (Lusignan 1990).

$\begin{array}{llc}\text { Cultivars } & \text { Cultivars } & \text { Total } \\ \text { précoces } & \text { tardifs } & \text { des cultivars }\end{array}$

Date de la fermeture du couvert végétal

Hauteur de la plante à la floraison

Longueur de la $2^{\mathrm{e}}$ feuille trifoliée le 6 juin 1990 en cm

Nombre de ramifications à la floraison

Nombre de ramifications à la maturité

Date de la 1 re fleur

Date de floraison

Nombre d'apothécies par $\mathrm{m}^{2}$ (Savarit, 1988)

Nombre d'apothécies par $\mathrm{m}^{2}$ (Lusignan, 1991)

Date de maturité des gousses

Hauteur de la plante à la maturité
$+0,09 \mathrm{NS}$

$+0,28 \mathrm{NS}$

$-0,22 \mathrm{NS}$

$+0,00 \mathrm{NS}$

$+0,08 \mathrm{NS}$

$+0,48 \mathrm{NS}$

$+0,38 \mathrm{NS}$

$-0,10 \mathrm{NS}$

$+0,57^{*}$

$+0,75^{\star \star}$
$+0,49$ NS

$-0,36 \mathrm{NS}$

$-0,37$ NS

$-0,19$ NS

$-0,07$ NS

$-0,45 \mathrm{NS}$

$-0,09$ NS

$-0,21 \mathrm{NS}$

$-0,51$ NS

$+0,24$ NS
$+0,11 \mathrm{NS}$

$+0,08 \mathrm{NS}$

$+0,88 \mathrm{NS}$

$+0,09$ NS

$-0,27$ NS

$+0,16 \mathrm{NS}$

$+0,32 \mathrm{NS}$

$-0,45 N S^{\star \star * *}$

$-0,15 \mathrm{NS}$

$+0,35 \mathrm{NS}$

$+0,54^{\star \star}$

* 13 degrés de liberté; ${ }^{\star \star} 15$ degrés de liberté; ${ }^{\star \star \star} 29$ degrés de liberté; ${ }^{\star \star \star \star} 5$ degrés de liberté. * Significatif $P<0,05 ;{ }^{\star \star}$ significatif $P<0,01$; NS: non significatif $P>0,05$.

sont le plus souvent résistantes. La moyenne d'attaque des 8 variétés à fleurs blanches est égale à $55 \%$ de plantes malades alors que la moyenne des 23 variétés à fleurs violettes correspond à $40 \%$ de plantes malades. Or le test de $t$ n'est pas significatif $(0,70>P>0,60)$. Par conséquent, les variétés à fleurs violettes ne sont pas significativement moins attaquées que les variétés à fleurs blanches.

\section{Caractères de la culture de soja n'étant pas liés à la sensibilité à la sclérotiniose}

\section{Sensibilité à la sclérotiniose et développement de la plante}

En 1988 à Savarit, nous avons montré qu'un couvert végétal dense favorise la sclérotiniose (Gondran, Leclercq, 1990). En effet, en maintenant l'humidité au sol, le couvert végétal favorise la formation des apothécies et par suite le développement de la maladie. On pourrait donc craindre que les différences variétales puissent être masquées par la date de fermeture du couvert végétal (interligne cessant de recevoir au sol la lumière directe au soleil). Cependant, le coefficient de corrélation entre le pourcentage de plantes malades et les dates de fermeture du couvert n'est pas significatif (tableau V). Par conséquent, le classement des obtentions suivant leur sensibilité n'est pas affecté par les dates différentes de fermeture du couvert végétal. Soulignons que ces dates correspondent à une interaction complexe entre notamment le développement des variétés, les dates de semis, la richesse du sol en fumure azotée et les disponibilités en eau du sol.

Pour approfondir cette constatation, nous avons étudié d'autres facteurs qui pourraient jouer sur la densité du couvert : longueur de la $2^{e}$ feuille trifoliée, hauteur de la plante à la floraison, nombre de ramifications à la floraison, nombre de ramifications à la maturité. Or nous constatons qu'aucun de ces caractères n'est lié au classement des cultivars pour la résistance à la sclérotiniose (tableau $\mathrm{V}$ ).

Nous pouvons dire que la sensiblité du soja à la sclérotiniose n'est influencée dans notre expérimentation ni par la date de fermeture du couvert, ni par d'autres caractères descriptifs du développement de la plante.

\section{Sensibilité à la sclérotiniose et apparition des fleurs}

L'apparition des fleurs de colza est très importante dans le développement de l'épidémie de sclérotiniose (Turkington et al, 1991).

Nous nous sommes demandé si la sensibilité à la sclérotiniose pouvait être liée à la date de floraison des variétés de soja et aux dates d'apparition de la première fleur. Le tableau $\mathrm{V}$ montre 
l'absence de corrélation de ces caractères avec le pourcentage de plantes malades notées sur les différents cultivars ou obtentions. Donc, à l'inverse des résultats constatés pour la culture de colza, nous n'avons pas mis en évidence l'influence de la floraison sur la sensibilité à la sclérotiniose des cultivars de soja.

\section{Sensibilité à la sclérotiniose et développement des apothécies}

Les apothécies sont émettrices d'ascospores, qui assurent l'infection des plantes. Nous avons donc pensé que certaines variétés pouvaient peut-être favoriser le développement des apothécies.

Nous avons donc compté le nombre des apothécies aux dates correspondant approximativement aux moments de leur plus forte sortie.

Ces dates étaient le 28 juillet 1988 à Savarit et le 10 juillet 1990 à Lusignan. À Savarit, les apothécies ont été dénombrées dans l'interligne large de $35 \mathrm{~cm}$ et long de $8 \mathrm{~m}$ (surface de $2,8 \mathrm{~m}^{2}$ ), correspondant à la ligne centrale de chaque parcelle. À Lusignan, les apothécies ont été comptées dans l'interligne sur une longueur de $20 \mathrm{~cm}$ de long de la ligne de $2 \mathrm{~m}$, où les sclérotes avait été disposées. Cela correspondait à une surface de $0,07 \mathrm{~m}^{2}$.

Nous avons calculé les coefficients de corrélation entre le pourcentage de plantes malades et le nombre d'apothécies par $\mathrm{m}^{2}$. Nous constatons (tableau V) qu'il n'y a aucune liaison entre ces 2 caractères. Par conséquent, à Lusignan comme à Savarit, les apothécies se formant dans chaque essai sont en nombre suffisant pour émettre des ascopores permettant de classer les variétés de soja suivant leur résistance à la sclérotiniose. En effet, il y a une bonne corrélation ( $r=0,81$ pour $5 \mathrm{dl}, P<0,05)$ entre le classement des 7 variétés installées à Savarit et des mêmes installées en 1990 à Lusignan.

En outre, nous pensons que les apothécies de toutes les parcelles de surface de $4,8 \mathrm{~m} 2$ de l'essai installé à Lusignan en 1990 forment un seul nuage d'ascopores. En effet, les parcelles qui n'ont pas d'apothécie, comme celles correspondant à "Weber", sont néanmoins fortement attaquées par la sclérotiniose.

\section{CONCLUSION ET DISCUSSION}

Nous avons pu mettre en évidence entre les variétés Labrador et Dom une différence significa- tive de résistance à la sclérotiniose de $64 \%$ de plantes malades et de $69 \%$ entre Kingsoy et Messidor. L'index de maladie utilisé par les chercheurs américains enregistre rarement de tels écarts (Nelson et al, 1991a). C'est grâce à la création et à l'utilisation d'une technique d'infection au champ par des sclérotes induits à la carpogenèse que nous avons pu réaliser une étude assez précise dans les conditions naturelles pour étudier la résistance du soja à $S$ sclerotiorum. Du fait de l'absence de cette technique, les auteurs américains n'ont pas réalisé suffisamment d'expérimentation au champ avant d'entreprendre des essais d'infection artificielle. C'est une des raisons pour lesquelles ils ne trouvent pas de corrélation significative entre les tests de laboratoire et les évaluations au champ (Nelson et al, 1991a). D'une façon plus générale, nous ne pouvons juger du comportement variétal du soja que si le niveau d'attaque moyen est élevé (ce que permet l'utilisation de notre protocole) et les conditions d'hygrométrie nécessaires à l'infection sont assurées durant une longue période, ce qui a pour effet de limiter les effets liés aux différences de précocité du matériel étudié.

La résistance que nous avons mise en évidence dans les conditions naturelles peut être partiellement expliquée par la hauteur de la plante à maturité et par la date de maturité des gousses. Lorsqu'on enlève ces effets, l'écart entre les variétés reste significatif, mais diminue à $43 \%$ entre Labrador et Dom. Par contre, il augmente à $71 \%$ entre Kingsoy et Messidor. Un caractère indépendant de la hauteur de la plante à maturité et de la date de maturité des gousses explique donc la résistance. Ce caractère n'est pas lié à la date de fermeture du couvert végétal, ni à d'autres nombreux caractères intervenant dans le développement de la plante. II ne semble être lié ni à la couleur des fleurs, ni à la date d'apparition de la première fleur, ni à la date de floraison. Or, certains auteurs (Favaron et al, 1988; Sutton et Deverall, 1984) ont montré que la plante de soja réagit à l'infection de nombreux pathogènes et notamment à celle de $S$ sclerotiorum par la production de glyceollines. Cette réaction cellulaire de défense n'a été mise en évidence que sur les cultivars Canton ou Lee. Nous n'avons aucune donnée sur la sensibilité à la sclérotiniose de Lee, mais nous savons que Canton a une sensibilité moyenne située entre celle de Kingsoy résistant et de Messidor sensible. II serait donc souhaitable de savoir si le classement des cultivars suivant la résistance à la sclérotiniose, établi par nos 
soins, pourrait être lié à la production de glyceollines. Si tel était le cas, l'aptitude de la plante à produire plus ou moins de cette phytoalexine expliquerait la résistance du soja à $S$ sclerotiorum.

\section{REMERCIEMENTS}

Nous remercions Mme MJ Villeger, de la firme Rustica-Semences, d'avoir mis à notre disposition des parcelles de soja infectées près de Montauban, d'avoir assuré semis et désherbage et de nous avoir aidé dans les notations.

Nous remercions MM A Pouzet et $Y$ Regnault du CETIOM, d'avoir mis à notre disposition des parcelles infectées par $S$ sclerotiorum à Savarit (17) et à Agen (47) ainsi que M G Arjaure et M Segura et leur équipe pour leur aide technique.

Nous remercions M G Laurens de la firme Protosem d'avoir mis à notre disposition des parcelles infectées par $S$ sclerotiorum à Villemur (31), ainsi que Mme $F$ Gaignard-Labalette et son équipe pour leur aide technique.

\section{RÉFÉRENCES}

Boland GJ, Hall R (1988) Epidemiology of sclerotinia stem rot of soybean in Ontario. Phytopathology 9, 1241-1245

Chun D, Kao LB, Lockwood JL, Isleib TG (1987) Laboratory and field assessment of resistance in soybean to stem rot caused by Sclerotinia sclerotiorum. Plant Dis 71, 811-815

Favaron F, Alghisi P, Marciano P, Magro P (1988) Polygalacturonase isoenzymes and oxalic acid produced by Sclerotinia sclerotiorum in soybean hypocotyls as elicitors of glyceollin. Physiol Mol Plant Pathol 33, 385-395
Ganya Al (1981) Principales mycoses du soja en Moldavie (en Russe). Mikol Fitopatol 15, 1, 37-43

Gondran J, Leclercq P (1990) La sclérotiniose du soja. Phytoma 423, 49-51

Gondran J, Leclercq P (1991) Pertes de rendements provoquées par la sclérotiniose du soja. ANPP. Proc $3^{\mathrm{e}}$ Conf Int Mal Plantes, Bordeaux (France), I, 303-310

Grau CR, Radke VL (1984) Effects of cultivars and cultural practices on sclerotinia stem rot of soybean. Plant Dis 68, 56-58

Li Hanching, Fu Chunyan (1981) Étude sur la sclérotiniose du soja (en chinois). Acta Phytopathol Sin 11, $3,20-24$

Lamarque C (1976) Éléments de biologie de Sclerotinia sclerotiorum sur tournesol en France. Inf Tech CETIOM 46, 21-25

Nelson BD, Helms TC, Kural I (1991a) Effects of temperature and pathogen isolate on laboratory screening of soybean for resistance to Sclerotinia sclerotiorum. Can J Plant Sci 71, 2, 347-352

Nelson BD, Helms TC, Olson MA (1991b) Comparison of laboratory and field evaluations of resistance in soybean to Sclerotinia sclerotiorum. Plant Dis 75 , 662-665

Penaud A (1991) Sclerotinia du soja : lutte chimique avant tout. Cultivar 300, 87-88

Purdy LH (1979) Sclerotinia sclerotiorum: history, diseases, symptomatology, host range, geographic distribution, and impact. Phytopathology 69, 875880

Sutton DC, Deverall BJK (1984) Phytoalexin accumulation during infection of bean and soybean by ascospores and mycelium of Sclerotinia sclerotiorum. Plant Pathol 33, 377-383

Turkington TK, Morrall RAA, Gugel RK (1991) Use of petal infestation to forecast sclerotinia stem rot of canola : evaluation of early bloom sampling 1985 1990. Can J Plant Pathol 13, 50-59 